\title{
Thermodynamics of a gauge-frustrated Kitaev spin liquid
}

\author{
T. Eschmann $\odot,{ }^{1, *}$ P. A. Mishchenko, ${ }^{2}$ T. A. Bojesen, ${ }^{2}$ Y. Kato, ${ }^{2}$ M. Hermanns,,${ }^{3,4}$ Y. Motome, ${ }^{2}$ and S. Trebst ${ }^{1}$ \\ ${ }^{1}$ Institute for Theoretical Physics, University of Cologne, D-50937 Cologne, Germany \\ ${ }^{2}$ Department of Applied Physics, The University of Tokyo, Tokyo 113-8656, Japan \\ ${ }^{3}$ Department of Physics, Stockholm University, AlbaNova University Center, SE-106 91 Stockholm, Sweden \\ ${ }^{4}$ Nordita, KTH Royal Institute of Technology and Stockholm University, SE-106 91 Stockholm, Sweden
}

(Received 25 January 2019; published 1 November 2019)

\begin{abstract}
Two- and three-dimensional Kitaev magnets are prototypical frustrated quantum spin systems, in which the original spin degrees of freedom fractionalize into Majorana fermions and a $\mathbb{Z}_{2}$ gauge field-a purely local phenomenon that reveals itself as a thermodynamic crossover at a temperature scale set by the strength of the bond-directional interactions. For conventional Kitaev magnets, the low-temperature thermodynamics reveals a second transition at which the $\mathbb{Z}_{2}$ gauge field orders and the system enters a spin-liquid ground state. Here, we discuss an explicit example that goes beyond this paradigmatic scenario- the $\mathbb{Z}_{2}$ gauge field is found to be subject to geometric frustration, the thermal ordering transition is suppressed, and an extensive residual entropy arises. Deep in the quantum regime, at temperatures of the order of one per mil of the interaction strength, the degeneracy in the gauge sector is lifted by a subtle interplay between the gauge field and the Majorana fermions, resulting in the formation of a Majorana metal. We discuss the thermodynamic signatures of this physics obtained from large-scale, sign-free quantum Monte Carlo simulations.
\end{abstract}

DOI: 10.1103/PhysRevResearch.1.032011

Introduction. In frustrated magnetism, lattice gauge theories are a ubiquitous tool to capture the physics of quantum spin liquids [1]. The fundamental distinction in these theories between confined and deconfined regimes corresponds to the formation of trivial, magnetically ordered states versus macroscopically entangled spin liquids. The principal nature of the underlying gauge theory can further be used to categorize different types of quantum spin liquids such as $\mathbb{Z}_{2}$ spin liquids [2,3], $U$ (1) spin liquids [4], or chiral spin liquids [5] - for which the corresponding gauge theory exhibits either a discrete $\mathbb{Z}_{2}$ or continuous $U(1)$ symmetry or an underlying Chern-Simons action [6]. This classification allows one to draw conclusions about the stability of the corresponding spin liquids, in particular, to thermal fluctuations. While the (spontaneous) breaking of time-reversal symmetry in chiral spin liquids mandates their thermal stability and the presence of a finite-temperature phase transition, a more complex picture emerges for $\mathbb{Z}_{2}$ and $U(1)$ spin liquids. Here, spatial dimensionality needs to be taken into account. For the $\mathbb{Z}_{2}$ spin liquid the elementary vison excitations of the underlying gauge structure are pointlike objects in two spatial dimensions, allowing them to proliferate at finite temperatures and destroy the entangled spin-liquid state. In contrast, in three dimensions the $\mathbb{Z}_{2}$ spin liquid is stable to thermal fluctuations, as now the visons form (small) looplike objects that cannot

\footnotetext{
*eschmann@thp.uni-koeln.de
}

Published by the American Physical Society under the terms of the Creative Commons Attribution 4.0 International license. Further distribution of this work must maintain attribution to the author(s) and the published article's title, journal citation, and DOI. destroy the spin liquid and break open into extended linelike objects only at a finite-temperature transition. For $U(1)$ spin liquids the elementary instanton excitations of the bare $U(1)$ gauge theory are pointlike objects in both two and three spatial dimensions, implying that these spin liquids are generically not stable at finite temperatures [7].

In this Rapid Communication, we consider the explicit example of a three-dimensional $\mathbb{Z}_{2}$ spin liquid, realized in a numerically tractable Kitaev model, that proves to be an exception from these paradigms. At sufficiently low temperature the gauge field is found to be subject to geometric frustration, arising from local constraints that impose a divergence-free condition and result in an extensive residual entropy. The net result is a suppression of the expected thermal ordering transition of the $\mathbb{Z}_{2}$ gauge field and the emergence of a spinliquid state that is "doubly frustrated," as it arises from the interplay of exchange frustration on the spin level and geometric frustration on the level of the emerging fractional degrees of freedom. Extensive quantum Monte Carlo simulations reveal that at ultralow temperatures of the order of one per mil of the interaction strength, i.e., deep in the quantum regime, the degeneracy in the gauge sector is lifted by a subtle interplay with the Majorana fermions, which emerge in parallel with the gauge field upon spin fractionalization. The formation of a collective ground state of these fermions, a Majorana metal with a distinct nodal-line structure, feeds back into the gauge sector and leads to the formation of columnar ordering of the gauge field. Our model system thereby proves to be a principal example of a spin liquid, for which not only the phenomenon of fractionalization, but also of the subsequent nontrivial interplay of the emergent fractional degrees of freedom and the underlying lattice gauge theory can be captured by numerically exact simulations. 
(a)

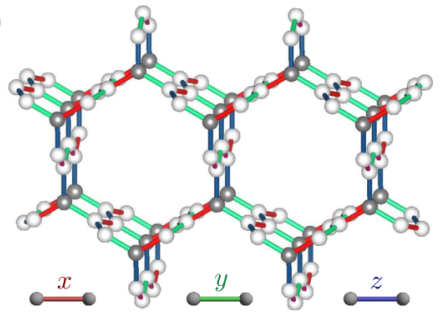

(b)

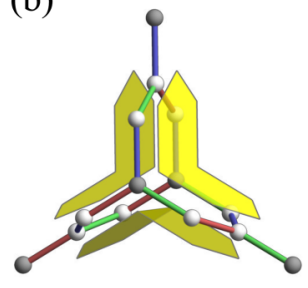

FIG. 1. The $(8,3) c$ lattice illustrated in (a) is a three-dimensional tricoordinated lattice, built around hexagonal sites (gray) interleaved with six zigzag chains. Its Schläfli symbol $(8,3) c$ indicates that all elementary plaquettes are of length 8 . Around each hexagonal site three such plaquettes meet as illustrated in (b).

Gauge frustration. The Kitaev model with its characteristic bond-directional spin exchanges of the form

$$
\mathcal{H}_{\text {Kitaev }}=\sum_{\langle j, k\rangle, \gamma} J_{\gamma} \sigma_{j}^{\gamma} \sigma_{k}^{\gamma}
$$

(with the Pauli matrices $\sigma^{\gamma}$ and $\gamma=x, y, z$ ) is well known to be analytically tractable for a class of two-dimensional (2D) $[8,9]$ and three-dimensional (3D) [10] lattices. The analytical treatment [8] relies on a parton construction that decomposes the spins into itinerant Majorana fermions and a static $\mathbb{Z}_{2}$ gauge field. The fact that the gauge field remains static and assumes, at sufficiently low temperatures, an ordered ground state is key for the exact solvability of the model, since it allows one to reduce the problem to one of free Majorana fermions hopping in a fixed background. In fact, a theorem by Lieb [11] describes the ground state of the gauge sector in terms of $\mathbb{Z}_{2}$ fluxes $W_{p}$ through the elementary plaquettesplaquettes of length $6,10, \ldots$ are flux-free $\left(W_{p}=+1\right)$, while plaquettes of length $4,8, \ldots$ carry a $\pi$ flux $\left(W_{p}=-1\right)$. Recent classification work of 3D Kitaev models [10] has shown that Lieb's theorem generically predicts the correct ground-state flux assignment, even for lattices that do not fulfill all its mathematical requirements. This is also true for the $(8,3) c$ lattice.

The key ingredient for the study at hand is a "frustrated" 3D lattice geometry whose central motif are hexagonal sites at which three plaquettes of length 8 meet (see the illustration in Fig. 1). Following the above intuition based on Lieb's theorem each of these plaquettes is destined to carry a $\pi$ flux, which conflicts with the fact that for any closed volume, such as the one spanned by the three plaquettes, the fluxes must obey a divergence-free condition-if a flux enters the volume through one of the plaquettes, it must leave through another one. The product of the three values of $W_{p}$ is thus fixed to +1 [10]. This condition allows only two of the three plaquettes to carry a $\pi$ flux and leaves one of the plaquettes in a flux-free state. For isotropic coupling strength $J_{x}=J_{y}=J_{z}$ this produces three possible flux arrangements per hexagonal site and an extensive residual entropy for the system. It is this formation of an extensive manifold of (almost) degenerate states in the gauge sector that designates the term "gaugefrustrated" for the Kitaev model at hand [12].

One way to relieve the frustration is to vary the relative coupling strengths. To see this, consider that every length- 8 plaquette consists of an uneven number of bond-directional

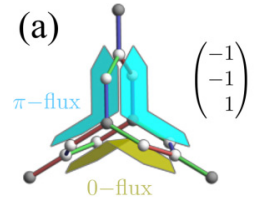

(d)
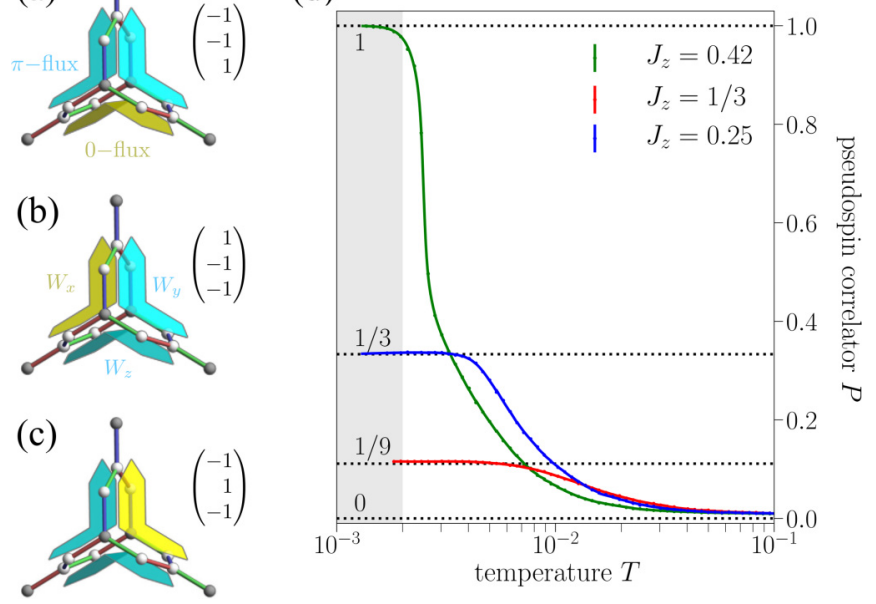

FIG. 2. Gauge frustration and pseudospins. (a)-(c) The 3-flux states that constitute the ground-state manifold of the $\mathbb{Z}_{2}$ gauge field. The plaquettes are colored cyan (yellow) to indicate a $\pi(0)$ flux. For each configuration the corresponding pseudospin vector is specified. (d) Pseudospin correlations as a function of temperature for different coupling strengths. Data shown are for system size $4 \times 4 \times 6$. The shaded area indicates the temperature region in which we have employed histogram reweighting techniques $[13,14]$ to extrapolate the data.

coupling types, e.g., $2 \times J_{z}$ for the bottom plaquette illustrated in Figs. 2(a)-2(c), while the two upper plaquettes have $3 \times J_{z}$ couplings. For $J_{z}>J_{x}=J_{y}$, one finds that the local threefold degeneracy is lifted and only one local gauge configuration, illustrated in Fig. 2(a), is favored. For $J_{z}<J_{x}=J_{y}$ the two flux configurations of Figs. 2(b) and 2(c) remain degenerate, thus only partially lifting the degeneracy.

To check that this phenomenon of gauge frustration indeed plays out in the model at hand, we have performed large-scale sign-free Monte Carlo simulations. To capture the local gauge physics, we define for any given hexagonal site a pseudospin vector

$$
\mathbf{W}=\left(\begin{array}{l}
W_{x} \\
W_{y} \\
W_{z}
\end{array}\right) \stackrel{(\mathrm{a})}{=}\left(\begin{array}{r}
-1 \\
-1 \\
1
\end{array}\right) \stackrel{(\mathrm{b})}{=}\left(\begin{array}{r}
1 \\
-1 \\
-1
\end{array}\right) \stackrel{(\mathrm{c})}{=}\left(\begin{array}{r}
-1 \\
1 \\
-1
\end{array}\right),
$$

where the individual components $W_{x, y, z}$ indicate the absence/presence of a $\pi$ flux in the three adjacent plaquettes. For the three states that fulfill the local divergence-free condition, their $\pi$ flux assignments are given on the right-hand side of the above equation in correspondence with Figs. 2(a)-2(c). For these pseudospin vectors we define a correlation function

$$
P=\frac{4}{3 N} \sum_{j}\left\langle\mathbf{W}_{0} \cdot \mathbf{W}_{j}\right\rangle,
$$

where 0 and $j$ denote two hexagonal sites. $P$ readily reveals the nature of the ground-state manifold and can be directly probed in our Monte Carlo simulations. Its expectation value is $P=1$ for the case of a single ground state of the gauge field $\left(J_{z}>J_{x}, J_{y}\right)$, and $P<1$ for the extensively degenerate cases, specifically $P=1 / 3$ for the local twofold degeneracy $\left(J_{z}<J_{x}, J_{y}\right)$ and $P=1 / 9$ for the local threefold degeneracy 
expected for isotropic coupling strengths $\left(J_{z}=J_{x}=J_{y}\right)$ (see the Supplemental Material [15]). Numerical results from Monte Carlo runs are shown in Fig. 2(d) for different strengths of $J_{z}$ [with $J_{x}=J_{y}=\left(1-J_{z}\right) / 2$ ]. The data clearly show that down to temperatures of the order of $10^{-2} J$ the pseudospin correlator goes to zero, indicating a completely disordered state of the gauge fields. At lower temperatures, the pseudospin correlator rises and eventually saturates. These simulations unambiguously confirm that the system indeed enters a regime of gauge frustration at low temperatures, with an extensive degeneracy building up in the gauge sector.

Lifting of gauge degeneracy. The formation of an "accidental" degeneracy, i.e., one that is not protected by any inherent symmetries of the system, is often accompanied by some residual effect that splits this degeneracy, at sufficiently small temperature scales, in favor of a unique (or less degenerate) ground state - an effect that typically goes hand in hand with a macroscopic phase transition. Such residual effects can include the energetic or entropic selection of ground states, driven either by otherwise negligible interactions (such as, e.g., longer-range interactions) or thermal fluctuations in an order-by-disorder scheme [16].

For the Kitaev system at hand, we find the particularly intriguing scenario that it is an (energetic) interplay between the emergent fractional degrees of freedom that ultimately lifts the gauge frustration discussed above. From the perspective of the itinerant Majorana fermions, the residual degeneracy in the gauge sector is equivalent to a complex scattering potential, as every individual gauge configuration corresponds to a distinct sign structure of the Majorana hopping amplitudes. In the gauge-frustrated regime, the collective state of the Majorana fermions is therefore best described as a thermal metal $[17,18]$, as the degeneracy in the gauge sector has a similar effect as (thermal) disorder. This observation points to a scenario where the formation of a collective Majorana state-a more conventional, disorder-free metallic state-might become favorable at the expense of inducing an ordering in the gauge sector. This is precisely what happens at ultralow temperatures, of the order of $10^{-3}$ of the magnetic coupling strength, in the system at hand-the itinerant Majorana degrees of freedom form a nodal-line semimetal, while simultaneously enforcing a columnar ordering in the gauge sector that lifts the gauge frustration. Schematically, the key signatures of these states are illustrated in Fig. 3, which shows the gapless nodal line in the Majorana band structure for different values of the exchange $J_{z}$, and the corresponding columnar ordering patterns of the gauge field.

Thermodynamics. To quantitatively probe this physics we have measured a variety of thermodynamic observables in quantum Monte Carlo (QMC) simulations covering four orders of magnitude in temperature. These QMC simulations are performed in the sign-free parton basis [19], i.e., we sample configurations of the gauge field, $\left\{u_{j k}= \pm 1\right\}$ for every bond $\langle j, k\rangle$ of the lattice, with the change of the Majorana free energy being calculated explicitly in every update step (either by exact diagonalization or a Green's function based kernel polynomial method $[20,21])$. This procedure also allows us to separately distill the entropic contributions to the specific heat of the Majorana fermions [22], as detailed in the Supplemental Material [15]. Results are given in Fig. 4(a) for the (a) $J_{z}=0.27$

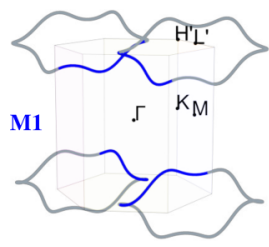

(b)

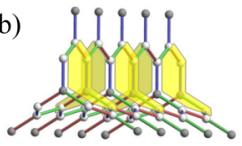

(c) $J_{z}=0.37$

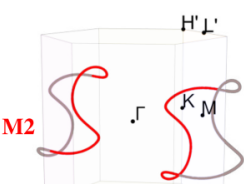

(d)

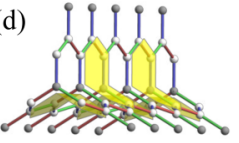

(e) $J_{z}=0.44$

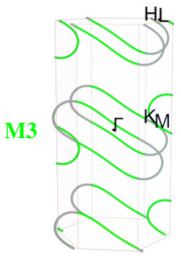

(f)

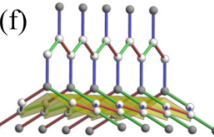

FIG. 3. Majorana semimetals and columnar gauge ordering. Evolution of the nodal line in the Majorana band structure for varying coupling $J_{z}$ (top row) calculated for the columnar-ordered gauge field configurations illustrated in the bottom row.

isotropic coupling point $\left(J_{x}=J_{y}=J_{z}\right)$. Some features of the multipeak structure of the specific heat are well known from conventional Kitaev models, such as the crossover feature at temperatures of order 1 , where the system releases about half of its entropy [see Fig. 4(b)] upon the fractionalization of the local spin degrees of freedom $[19,23]$, primarily by the Majorana fermions (whose energy scale is set by the magnetic coupling strength). Below this crossover there are two additional features At a temperature of about $10^{-2}$ a
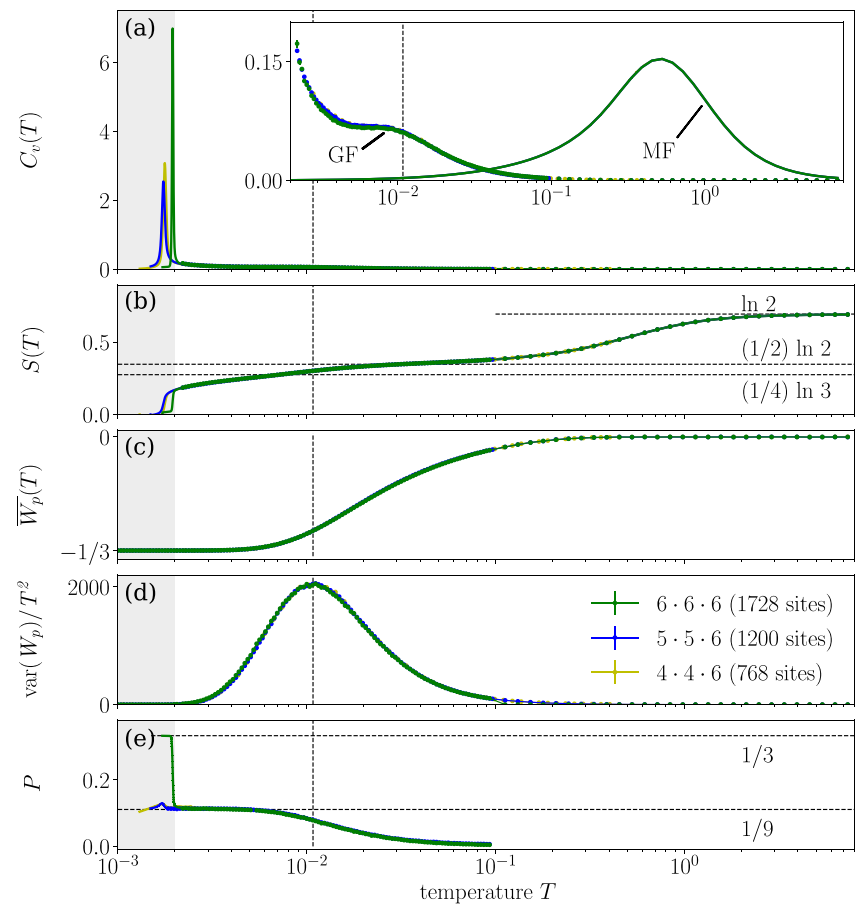

FIG. 4. Thermodynamic signatures for the isotropic system. (a) Specific heat, separated into contributions of $\mathbb{Z}_{2}$ gauge field (GF) and itinerant Majorana fermions (MF). (b) Entropy per spin. (c) Flux per plaquette. (d) Fluctuation of the flux per plaquette. (e) Pseudospin correlator (3). The dashed line indicates the temperature scale at which the system enters the constrained manifold, corresponding to the maximum in the flux fluctuations and a residual entropy of $(1 / 4) \ln 3$. Error bars are smaller than the symbol sizes. 
broad shoulder forms, which does not show any scaling with system size pointing to a local crossover phenomenon. It is at this temperature scale that the system enters the manifold of "gauge-frustrated" states, which is evident from (i) the average plaquette flux dropping to a value of $\left\langle W_{p}\right\rangle=-1 / 3=$ $(-2+1) / 3$ (expected for local configurations where two out of three plaquettes have a $\pi$ flux, i.e., $W_{p}=-1$, and one plaquette remains flux-free, $W_{p}=+1$ ), (ii) the fluctuations of the plaquette flux exhibiting a maximum upon entering this constrained ground-state manifold, and (iii) the pseudospin correlator (3) raising and saturating at the expected value of $P=1 / 9$, as documented in Figs. 4(c)-4(e). Below this second crossover peak, at a temperature of the order of $2 \times 10^{-3}$, one finds a sharp peak in the specific heat that sharpens with increasing system size - this is a true thermal phase transition, where the system releases entropy by forming a columnar ordering of the gauge field. In this ordered state every column of hexagonal sites exhibits a staggered pattern of the flux-free plaquettes as indicated by the yellow plaquettes in Fig. 3(b). However, since the columns order individually and there are two possible staggered states for each column, the resulting overall order not only still allows for a residual entropy, but also breaks the lattice rotational symmetry. This is a remarkable symmetry-breaking effect as it plays out solely in the gauge sector.

Phase diagram. Expanding this analysis of key thermodynamic observables to a range of $J_{z}$ parameters, we have compiled the composite phase diagram of Fig. 5. Plotted here are different indicators for the crossover scale to the constrained gauge manifold. As proxies for this crossover, we have marked (i) the location of the peak in the variance of the fluxes, akin to Fig. 4(d), by the solid green circles and (ii) the line of temperature points at which the pseudospin correlator (3) crosses $P=1 / 9$ by the solid white circles. The low-temperature phase transition, at which the concurrent formation of a nodal-line Majorana semimetal and columnar order of the gauge field occurs, is indicated by the white squares. Depending on the strength of $J_{z}$, we distinguish two principal scenarios. First, there is a line of transitions (indicated by the solid white squares) where it is the formation of the Majorana metal that lifts the degeneracy in the gauge sector and enforces the columnar gauge order. This is the case for $J_{z} \leqslant 1 / 3$. For $J_{z} \gtrsim 0.40$, it is the energetics within the gauge sector that readily selects a single configuration of the constrained gauge field for each hexagonal site [see Fig. 2(a)], which results in the columnar ordering depicted in Fig. 3(f). For $1 / 3<J_{z} \lesssim 0.40$, a more subtle mechanism is at play where the energetics of the gauge field favors the same type of columnar order as for $J_{z} \gtrsim 0.40$, but the minimization of the Majorana energy enforces yet another type of columnar order, depicted in Fig. 3(d), which, in a certain sense, is an intermediate type of order with a staggered flux pattern involving flux-free states on some of the bottom $W_{z}$ plaquettes. The phase diagram thus reveals multiple distinct regimes, in which a subtle interplay between the emergent parton degrees of freedom leads to the formation of different types of collective ground states-including gapless spin liquids with a Majorana nodal line and columnar-ordered $\mathbb{Z}_{2}$ gauge fields.

Conclusions. The main results of the Rapid Communication at hand are two advances in the conceptual understanding

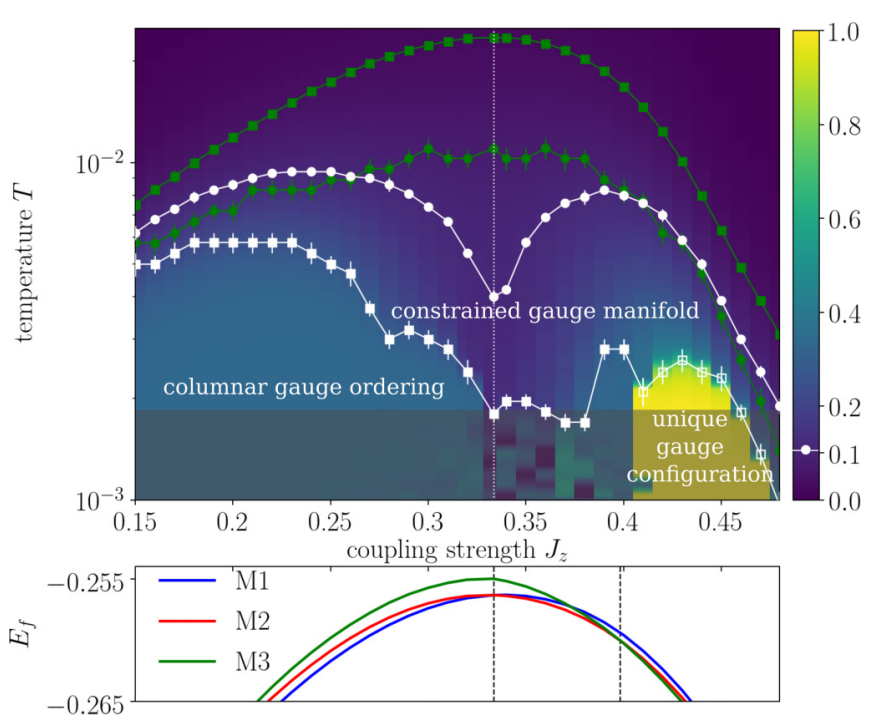

FIG. 5. Finite-temperature phase diagram. The background contour plot indicates the pseudospin correlations (3) as a function of temperature $T$ and coupling strength $J_{z}$. The crossover scale at which the system enters the flux constrained manifold is indicated by (i) solid green circles indicating the peak in the variance of the fluxes and (ii) solid white circles indicating temperature points for which the pseudospin correlator $P=1 / 9$. The onset of flux ordering is signaled in the high-temperature regime where we mark the line along which the flux becomes $\left\langle W_{p}\right\rangle=-1 / 6$ (green squares). The low-temperature columnar ordering transition of the gauge field is marked by the white squares. Solid squares indicate transitions where the degenerate manifold of constrained gauge configurations is lifted by the formation of a Majorana metal, while open squares indicate transitions driven by an energetic selection within the gauge sector. Data shown are for system size $4 \times 4 \times 6$. The lower panel shows the ground-state energy of the Majorana metals for the three competing types of gauge ordering, using the color code of Fig. 3.

of quantum spin liquids. First, we have introduced the concept of "gauge frustration," which we showcased in a 3D Kitaev model where the emergent $\mathbb{Z}_{2}$ gauge degrees of freedom are subject to local constraints resulting in an extensive residual entropy. Second, we showed by large-scale numerical simulations that this residual entropy can be lifted by an interplay of the $\mathbb{Z}_{2}$ lattice gauge theory and the itinerant Majorana fermions, which concurrently emerge with the gauge field upon fractionalization of the original local spin degrees of freedom. As such, the model at hand realizes a scenario intermediate between more conventional Kitaev models where the parton degrees of freedom fully decouple (allowing for an analytical solution where one first identifies the ground state of the gauge field and subsequently solves the Majorana problem), and the scenario of strongly interacting partons as it is the case for, e.g., a $U(1)$ spin liquid, in which the gauge field remains heavily fluctuating to the lowest temperatures and thereby strongly feeds back into the formation of a collective parton state.

Acknowledgments. T.E., M.H., and S.T. acknowledge partial funding by the Deutsche Forschungsgemeinschaft (DFG, German Research Foundation) Projektnummer 277146847SFB 1238 (Projects $\mathrm{C} 02$ and C03). M.H. acknowledges partial funding by the Knut and Alice Wallenberg Foundation and 
the Swedish Research Council. P.A.M, T.A.B., Y.K., and Y.M. acknowledge funding by Grant-in-Aid for Scientific Research under Grants No. 15K13533 and No. 16H02206. Y.M. and
Y.K. were also supported by JST CREST (JPMJCR18T2). The numerical simulations were performed on the JUWELS cluster at the Forschungszentrum Jülich.
[1] L. Savary and L. Balents, Quantum spin liquids: A review, Rep. Prog. Phys. 80, 016502 (2017).

[2] N. Read and S. Sachdev, Large- $N$ Expansion for Frustrated Quantum Antiferromagnets, Phys. Rev. Lett. 66, 1773 (1991).

[3] T. Senthil and M. P. A. Fisher, $Z_{2}$ gauge theory of electron fractionalization in strongly correlated systems, Phys. Rev. B 62, 7850 (2000).

[4] P. W. Anderson, Resonating valence bonds: A new kind of insulator? Mater. Res. Bull. 8, 153 (1973).

[5] V. Kalmeyer and R. B. Laughlin, Equivalence of the Resonating-Valence-Bond and Fractional Quantum Hall States, Phys. Rev. Lett. 59, 2095 (1987).

[6] X.-G. Wen, Quantum orders and symmetric spin liquids, Phys. Rev. B 65, 165113 (2002).

[7] Possible exceptions are discussed in the context of quantum spin ice [24].

[8] A. Kitaev, Anyons in an exactly solved model and beyond, Ann. Phys. 321, 2 (2006).

[9] M. Hermanns, I. Kimchi, and J. Knolle, Physics of the Kitaev model: Fractionalization, dynamic correlations, and material connections, Annu. Rev. Condens. Matter Phys. 9, 17 (2018).

[10] K. O'Brien, M. Hermanns, and S. Trebst, Classification of gapless $\mathbb{Z}_{2}$ spin liquids in three-dimensional Kitaev models, Phys. Rev. B 93, 085101 (2016).

[11] E. H. Lieb, Flux Phase of the Half-Filled Band, Phys. Rev. Lett. 73, 2158 (1994).

[12] A similar scenario of "gauge frustration" plays out in the 3D Kitaev model on the hypernonagon lattice [25] where the pattern of $\pm \pi / 2$ fluxes arising from elementary 9-bond loops is subject to geometric frustration.

[13] A. M. Ferrenberg and R. H. Swendsen, New Monte Carlo Technique for Studying Phase Transitions, Phys. Rev. Lett. 61, 2635 (1988).

[14] A. M. Ferrenberg and R. H. Swendsen, Optimized Monte Carlo Data Analysis, Phys. Rev. Lett. 63, 1195 (1989).
[15] See Supplemental Material at http://link.aps.org/supplemental/ 10.1103/PhysRevResearch.1.032011 for details on the lattice structure, pseudospin correlations and the quantum Monte Carlo simulations applied in this work.

[16] J. Villain, R. Bidaux, J.-P. Carton, and R. Conte, Order as an effect of disorder, J. Phys. France 41, 1263 (1980).

[17] J. T. Chalker and P. D. Coddington, Percolation, quantum tunneling and the integer Hall effect, J. Phys. C: Solid State Phys. 21, 2665 (1988).

[18] J. T. Chalker, N. Read, V. Kagalovsky, B. Horovitz, Y. Avishai, and A. W. W. Ludwig, Thermal metal in network models of a disordered two-dimensional superconductor, Phys. Rev. B 65, 012506 (2001).

[19] J. Nasu, M. Udagawa, and Y. Motome, Vaporization of Kitaev Spin Liquids, Phys. Rev. Lett. 113, 197205 (2014).

[20] A. Weiße, Green-Function-Based Monte Carlo Method for Classical Fields Coupled to Fermions, Phys. Rev. Lett. 102, 150604 (2009).

[21] P. A. Mishchenko, Y. Kato, and Y. Motome, Finite-temperature phase transition to a Kitaev spin liquid phase on a hyperoctagon lattice: A large-scale quantum Monte Carlo study, Phys. Rev. B 96, 125124 (2017)

[22] Y. Shimomura, S. Miyahara, and N. Furukawa, Frustrationinduced dodecamer ordering in the double-exchange spin ice model on the kagome lattice, J. Phys. Soc. Jpn. 74, 661 (2005).

[23] J. Nasu, M. Udagawa, and Y. Motome, Thermal fractionalization of quantum spins in a Kitaev model: Temperature-linear specific heat and coherent transport of Majorana fermions, Phys. Rev. B 92, 115122 (2015).

[24] L. Savary and L. Balents, Spin liquid regimes at nonzero temperature in quantum spin ice, Phys. Rev. B 87, 205130 (2013).

[25] Y. Kato, Y. Kamiya, J. Nasu, and Y. Motome, Chiral spin liquids at finite temperature in a three-dimensional Kitaev model, Phys. Rev. B 96, 174409 (2017). 